\title{
Implementation of Xpert MTB/RIF for routine point-of-care diagnosis of tuberculosis at the primary care level
}

\author{
Kate Clouse, Liesl Page-Shipp, Heather Dansey, Bridgette Moatlhodi, Lesley Scott, Jean Bassett, \\ Wendy Stevens, Ian Sanne, Annelies Van Rie
}

Xpert MTB/RIF (Xpert) offers rapid detection of Mycobacterium tuberculosis and rifampicin resistance. However, little is known about routine point-of-care (POC) use in high TB/HIV burden settings. We describe our experiences of launching Xpert as the POC, initial diagnostic for all TB suspects at a primary healthcare clinic in Johannesburg, South Africa. Noted important benefits of POC Xpert were fewer clinic visits, rapid detection of TB and rifampicin resistance, real-time assessment of accompanying household members of new TB cases, and increased staff motivation for TB screening.

While Xpert results are available within 2 hours, actual turnaround time was longer for most patients because of sample preparation time and clinic congestion. Consequently, a GX4 instrument did not result in a 16-test capacity during an 8-hour working day, and some patients did not receive same-day results. Loss to follow-up was an unforeseen challenge, overcome by clinic flow changes, marking of clinic files, documenting patients' physical description and locating patients in the clinic by cell phone. Staff with high school education successfully performed the assay after minimal training.

Human resource requirements were considerable, with a minimum of 2 staff needed to supervise sputum collection, process sputum, perform assays, and document results for an average of 15 TB suspects daily. POC placement of the instrument transferred logistical responsibilities to the clinic, including quality assurance, maintenance, stock control and cartridge disposal.

POC use of Xpert is feasible at the primary healthcare level but must be accompanied by financial, operational and logistical support.

S Afr Med J 2012;102(10):805-807. DOI:10.7196/SAMJ.5851
In December 2010, the WHO endorsed Xpert MTB/RIF (Cepheid, Sunnyvale, CA, USA) as a first-line diagnostic for TB in patients suspected of HIV-associated TB or multidrug-resistant (MDR) TB. ${ }^{1}$ South Africa, the first country to roll out the assay, opted to place the instruments at centralised microscopy centres and reference

Clinical HIV Research Unit, University of the Witwatersrand, Johannesburg Kate Clouse, $\mathrm{PhD}, \mathrm{MPH}$

Ian Sanne, MB BCh, FCP (SA), FRCP (Lon), DTM\&H

Department of Epidemiology, University of North Carolina Gillings School of Global Public Health, Chapel Hill, NC, USA

Kate Clouse, PhD, MPH

Annelies Van Rie, MD, PhD

Right to Care, Johannesburg

Liesl Page-Shipp, MB BCh, MPH

Bridgette Moatlhodi

Ian Sanne, MB BCh, FCP (SA), FRCP (Lon), DTM\&H

Witkoppen Health and Welfare Centre, Johannesburg Heather Dansey, MB BCh

Jean Bassett, MB BCh

National Health Laboratory Services, Johannesburg

Lesley Scott, $\mathrm{PhD}$

Wendy Stevens, MB BCh, MMed (Haem), FCPath (Haem)

Department of Molecular Medicine and Haematology, School of Pathology, University of the Witwatersrand

Lesley Scott, $\mathrm{PhD}$

Wendy Stevens, MB BCh, MMed (Haem), FCPath (Haem) laboratories of the National Health Laboratory Service, mainly owing to cost considerations and to allow rapid roll-out. ${ }^{2}$

Xpert MTB/RIF (Xpert)'s short turnaround time and simplicity raised potential for point-of-care (POC) use., The motivation for POC technology (be it HIV, CD4 or TB testing) is to provide sameday results, hasten treatment initiation, and avoid loss to follow-up during the diagnostic process. Little is known about POC use of Xpert at primary healthcare level, and operational research is needed before its use at the peripheral level can be recommended. ${ }^{3,5}$

In July 2011, we launched Xpert as the initial, routine, POC diagnostic for all TB suspects at Witkoppen Health and Welfare Centre, a high-volume (8 500 monthly visits), NGO-operated primary care clinic. The centre serves residents of informal settlement areas within northern Johannesburg that have a high HIV and TB burden.

\section{Human resource requirements}

Human resource requirements were greater than anticipated, because the use of Xpert increased the number of TB cases identified and because staff time per TB suspect assessed was higher than expected. While Xpert is in principle a 2-hour test, ${ }^{6}$ this only refers to the assay turnaround time. Registering clients in the TB suspect register, educating them on providing a quality sputum, adding the buffer, processing the sample, transferring the sample into the cartridge, and transporting the cartridge to the instrument, all require personnel and time. Once the assay is ready, the results must be printed and filed and the patient traced within the clinic and referred to the next clinic service, depending on the Xpert result and reason for visit.

Consequently, while we expected to need 1 full-time employee, we found we needed a minimum of 2 full-time employees to ensure operation of POC Xpert services for an average of $16 \mathrm{~TB}$ suspects (range: 7 - 29) daily. The clinic opted to employ 2.5 full-time staff to ensure continuous POC Xpert service: 1 person at the outdoor sputum booth for patient assistance, sample buffering and data entry in the suspect register; 1 in the Xpert instrument room to perform the 
assay, communicate results to patients and healthcare workers and capture data in clinic file and TB registers; and a third person at 50\% effort for assistance during the busy afternoon period and to cover for lunch breaks and absences. In addition, 1 low-skilled runner carries buffered sputum samples between the outdoor sputum collection booth and the instrument room and traces patients within the clinic.

This 'POC Xpert team' was employed in addition to the existing clinic TB team, which consists of 1 part-time physician, 1 fulltime nurse and 2 full-time auxiliary nurses. Specialised skills were not required, as staff with high school education and no formal laboratory training performed the Xpert assay after 2 days of training.

\section{Instrument requirements}

According to the WHO, the four-module Xpert instrument has a capacity of $15-20$ tests per working day. ${ }^{7}$ While this is probably a correct estimate for instruments placed in a laboratory, 2 fourmodule instruments were required to process an average of 15 samples daily at POC. Even though the clinic operates 8 hours daily, the first sputum sample is only available 2 - 3 hours after the first TB suspect arrives at the clinic, as clients proceed through several administrative and care steps before sputum is collected. The last time a cartridge can be loaded and provide a same-day result is 2 or preferably 3 hours before the clinic closes. Tests for patients who present late in the day are run overnight and results are available the following day. In addition, tests resulting in errors or invalid results need to be repeated. We observed an error or invalid rate of 3.4\%, consistent with the $2.4-5.9 \%$ observed elsewhere. ${ }^{8,9}$

\section{Infrastructure requirements}

TB testing at POC, outside of the laboratory, requires emphasis on infection control and biosafety. An outdoor sputum booth was constructed to provide adequate ventilation, and this is where sputum collection is performed and the Xpert buffer added. Clinic staff are instructed to wear N-95 masks when collecting and buffering sputum, and when communicating face-to-face with TB suspects. New TB cases are asked to wear surgical masks. Conditions for instrument operation within the clinic were also optimised, ensuring a temperature $<30^{\circ} \mathrm{C}$, stable electrical supply, connection of the 2 instruments to 1 computer, security measures against theft, and adequate storage space for the cartridges.

\section{Changing the clinic flow and optimising patient retention}

In contrast to our expectations, many patients initially failed to receive their Xpert result because they had left the clinic or were untraceable within the clinic 2 hours after the cartridge was placed in the instrument. To overcome this problem many interventions requiring co-operation from the entire clinic have been implemented.

First, a paper slip identifying the TB suspect as receiving an Xpert assay is stapled to the outside of the clinic file and only removed once the patient receives the result. This allows the patient to attend other clinic services while the assay is running, and prevents the clinic from unknowingly checking out the patient before they receive their results. It also serves to alert the clinician that the patient has been identified as a TB suspect and is currently awaiting Xpert results. Second, the TB suspect's cell phone number is recorded (when available) and the number is tested on the spot to ensure it is correct and the phone is turned on. When the test is completed, a text message is sent: 'Your TB results are ready. Please come to the TB Room.' Third, a physical description of each TB suspect is recorded, including the colour of clothing worn. When the TB suspect cannot be reached by phone, runners are deployed to help locate patients in the clinic using this description.

Since these measures were implemented, TB suspects have received their test result on the same day, except for those who need to leave the clinic for work purposes and those who provide a sputum sample $<2$ hours before the clinic closes.

The aim of rapid POC technology is to receive a same-day test result and to act upon it in real-time. When diagnosis is based on smear microscopy, TB suspects provide a sputum sample and come back a few days later. At the second visit, they arrive in the morning and have results ready in their file, and the nurse or clinician can start TB treatment immediately. In contrast, when using Xpert at POC, new cases of TB are identified throughout the day. Staff must therefore be ready to register a new case and initiate treatment at any time, even at the end of the workday.

Furthermore, when provided with Xpert at POC, TB suspects continue their clinic visit while the sample is being processed. However, the results of the Xpert assay may not yet be ready, creating the need for 2 consultations on the same day, once before and once after the Xpert results are available. Since the vast majority of TB suspects are Xpert negative, the clinic opted to presumptively manage TB suspects as Xpert negative and schedule a follow-up visit 1 week later. Consequently, only clients with a positive Xpert result may need to be seen twice by a healthcare worker on a single day or traced to begin TB treatment.

\section{Shifting responsibilities from laboratory to clinic}

Positioning Xpert instruments at POC in the clinic has management and cost implications beyond the purchase of the instrument and cartridges required for processing samples. Increased overhead and management costs include instrument insurance, decentralised billing, cartridge procurement and stock control, and data management to ensure results are linked to a national TB database. POC laboratories also require consistent electricity, regular maintenance and calibration of the instruments, staff training, certification, and participation in external quality assurance programmes.

\section{Anticipated and unanticipated benefits of POC Xpert}

As expected, placing Xpert at POC resulted in increased case detection, same-day treatment initiation in over $80 \%$ of new cases, and knowledge of the M. tuberculosis strain's susceptibility to rifampicin on the day that treatment is started.

In addition, we discovered some unanticipated benefits. Clinic staff were enthusiastic about the possibility of a same-day result, which increased their motivation to identify TB suspects. Clinicians especially valued the ability to rapidly assess rifampicin resistance among patients not responding to treatment. Awareness of on-site rapid TB testing led clinic staff to request a TB test for themselves when symptomatic. Additionally, the same-day availability of results enabled real-time TB contact identification when a new case was accompanied by a partner or relatives.

\section{Conclusion}

While our results were overwhelmingly positive, our experiences highlight that the infrastructure, instrument and human resource 
requirements for POC positioning of Xpert exceeded expectations. As a result, the responsibilities transferred from laboratory to clinic went beyond sample processing. The magnitude of the challenges will be highly dependent on the existing infrastructure and current organisation of TB diagnosis and treatment facilities. Programmes will therefore need to carefully weigh the benefits against infrastructural and human resource needs when deciding on a POC or laboratory policy for implementation of Xpert MTB/RIF.

Financial disclosure. This work was supported by the United States President's Emergency Plan for AIDS Relief (PEPFAR) and National Institutes of Health (NIH) grant UM1 AI069463; the United States Agency for International Development in a grant to Right to Care 674-A00-08-00007; and the NIH Fogarty International Center's International Clinical, Operational and Health Services Research Training Award (ICOHRTA) AIDS/TB grant U2RTW007370. The funders had no role in study design, data collection and analysis, decision to publish, or preparation of the manuscript. The authors declare that they have no conflicts of interest.
References

1. World Health Organization. Roadmap for rolling out Xpert MTB/RIF for rapid diagnosis of TB and MDR-TB. Geneva: 8 Dec 2010. http://www.who.int/tb/laboratory/roadmap_xpert_mtb-rif.pd (accessed 22 March 2012)

2. Meyer-Rath G, Bistline K, Long L, et al. The incremental cost of introducing Xpert MTB/RIF into the South African national tuberculosis programme: Results of the National TB Cost Model 2011/12 2016/17. Health Economics and Epidemiology Research Office. July 2011. http://www.bu.edu/cghd files/2011/07/HERO_Policy_Brief_No_1-National_TB_Cost_Model_South_Africa_July_20111.pd (accessed 22 March 2012).

3. Van Rie A, Page-Shipp L, Scott L, Sanne I, Stevens W. Xpert MTB/RIF for point-of-care diagnosis of TB in high-HIV burden, resource-limited countries: hype or hope? Expert Rev Mol Diagn 2010;10(7):937 946

4. Small PM, Pai M. Tuberculosis diagnosis - time for a game change. N Engl J Med 2010;363(11):10701071.

5. Trébucq A, Enarson DA, Chiang CY, et al. Xpert MTB/RIF for national tuberculosis programmes in low-income countries: when, where and how? Int J Tuberc Lung Dis 2011;15(12):1567-1572.

6. Cepheid. Xpert MTB/RIF brochure (CGXMTB/RIF-10; 300-7810 Rev A). (Apr 2009). http:// tbevidence.org/documents/rescentre/sop/XpertMTB_Broch_R9_EU.pdf (accessed 22 March 2012).

7. World Health Organization. Rapid implementation of the Xpert MTB/RIF diagnostic test. 2011. http: whqlibdoc.who.int/publications/2011/9789241501569_eng.pdf (accessed 22 March 2012).

whilibdoc.who.int/publications/2011/9789241501569_eng.pdf (accessed 22 March 2012). 8. Boehme CC, Nicol MP, Nabeta P, et al. Feasibility, diagnostic accuracy, and effectiveness o
decentralised use of the Xpert MTB/RIF test for diagnosis of tuberculosis and multidrug resistance: decentralised use of the Xpert MTB/RIF test for diagnosis of tubercu,
multicentre implementation study. Lancet 2011;377(9776):1495-1505.

9. Scott LE, McCarthy K, Gous N, et al. Comparison of Xpert MTB/RIF with other nucleic acid technologies for diagnosing pulmonary tuberculosis in a high HIV prevalence setting a prospective .

\title{
Determinants of mortality in Nigerian children with severe anaemia
}

\author{
Samuel Adegoke, Adenike Ayansanwo, Isaac Oluwayemi, John Okeniyi
}

Background. Severe anaemia (haemoglobin concentration $<50 \mathrm{~g} / \mathrm{l}$ ) is a major cause of paediatric hospital admissions and deaths in the tropics.

Objectives.To examine the pattern and predictors of mortality among severely anaemic children.

Methods. A prospective cross-sectional study was conducted among children with severe anaemia at the Children's Emergency Room of the University Teaching Hospital, Ado-Ekiti, Nigeria. Sociodemographic characteristics, clinical features, laboratory findings and co-morbidities of the survivors and those who died were compared by logistic regression analysis.

Results. Of the 1735 children admitted, 311 (17.9\%) had severe anaemia, with a case-fatality rate of $9.3 \%$. The presence of respiratory distress (95\% confidence interval (CI) $2.1-3.6, p=0.031$ ); acidosis (95\% CI 1.8 - 2.7, $p=0.010$ ); coma (95\% CI $0.1-0.3, p=0.001$ ); hypotension ( $95 \%$ CI $2.0-4.2, p=0.020$ ); and bacteraemia (95\% CI 3.1 - 3.9; $p=0.008)$ were the significant independent predictors of death with regression analysis.

Conclusion. Early recognition with prompt and appropriate anticipatory intervention is essential to reduce mortality from severe anaemia.

S Afr Med J 2012;102(10):807-810. DOI:10.7196/SAMJ.5240
Severe anaemia is a major cause of paediatric hospital admissions and deaths in many African countries, with a hospital prevalence of $10-30 \%$ and case fatality rates between 4 and $10 \% .^{1,2}$ Anaemia, in

Department of Paediatrics and Child Health, Obafemi Awolowo University, Ile-Ife, Nigeria

Samuel Adegoke, MB ChB, MPH, FWACP

John Okeniyi, BSc, MB ChB, FWACP

Department of Haematology, University Teaching Hospital, Ado-Ekiti, Nigeria Adenike Ayansanwo, MB BS, FMC Path

Department of Paediatrics, University Teaching Hospital, Ado-Ekiti, Nigeria Isaac Oluwayemi, MB BS, FWACP which blood oxygen carrying capacity is reduced, is characterised by a low haemoglobin concentration. ${ }^{3}$ The World Health Organization (WHO) defined severe anaemia in patients with malaria as a haemoglobin concentration less than $50 \mathrm{~g} / 1 .{ }^{3}$ Anaemia becomes lifethreatening when affected individuals develop complications such as heart failure, metabolic acidosis and hypoxic encephalopathy. ${ }^{4}$

Severe falciparum malaria remains the leading cause of paediatric anaemia in sub-Saharan Africa. ${ }^{1,2,4}$ Anaemia may develop in children already debilitated by other conditions such as nutritional deficiencies, bacteraemia, human immunodeficiency viral (HIV) infections, haemoglobinopathy, glucose-6-phosphate dehydrogenase (G6PD) deficiency, hookworm infestation and leukaemia. In most cases, these factors tend to co-exist. ${ }^{1}$

Severe anaemia-related deaths continue to occur in many centres in the tropics, even where blood transfusion services are readily available. ${ }^{1,2}$ To limit this mortality, we studied children admitted with severe anaemia in a newly established tertiary health centre 